\section{Choroidal Ischemia after Carotid Artery Stenting for Cerebral Infarction: A Case Report}

\section{Dear Editor,}

Choroidal ischemia is an uncommon ocular disorder caused by various systemic diseases or local vascular disorders. Atheromatous lesions (by producing emboli), stenosis, or occlusion can be considered carotid artery-related causes that lead to acute choroidal ischemia [1]. Carotid artery stenting (CAS) is the mainstay treatment for carotid stenosis. However, CAS is restricted in cases with a high chance of emboli shedding into the distal vascular bed because plaque manipulation during the CAS procedure can generate such emboli [2]. Cases of choroidal ischemia caused by emboli occurring after CAS are rare, and few have been reported. Hence, we present a rare case of choroidal ischemia immediately after the CAS procedure.

A 60-year-old female patient was hospitalized for cerebral infarction and underwent CAS for severe left proximal internal carotid artery stenosis. On CAS procedure day, the patient experienced visual disturbances, including a visual field defect in the left eye, and she was referred to the ophthalmology department. On examination, the visual activity was finger counting at $10 \mathrm{~cm}$ and $7 \mathrm{mmHg}$ intraocular pressure in the left eye. Slit-lamp examination showed a clear lens and anterior chamber state without new vessels on the iris. A characteristic triangular patch (Amalric sign) merging into a well-defined atrophic area of the retinal pigment epithelium was observed in the eye with choroidal ischemia. Hyperpigmented grouped lines appeared over non-perfused choroidal areas (Siegrist streaks). Circumscribed ischemia of smaller choroidal arterioles and capillaries appears as multifocal yellow lesions in the posterior fundus (Elschnig spot) [3]. Fundus exam-

Received: September 10, 2021 Final revision: November 18, 2021 Accepted: November 18, 2021 ination showed an edematous disc with blurred margins and the outer retinal ischemic changes in the nasal and temporal areas (associated with the choroidal ischemia). Amalric signs and Elschnig spots are also well presented in the images (Fig. 1A). Fluorescence angiography demonstrated choroidal hypoperfusion in the corresponding area (Fig. 1B). After a week, visual acuity improved to $20 / 125$, and fundus examination revealed a less edematous and slightly pale disc and decreased the outer retinal ischemic changes in the nasal and temporal regions compared to those in the previous examination (Fig. 1C). Indocyanine green angiography revealed a remarkable delay in the choroidal filling time and a wide area of patchy choroidal filling defects (Fig. 1D). These patchy hypofluorescence areas were considered to indicate that the short posterior ciliary artery (PCA) supplying the area was embolized and became ischemic. After 15 months, visual acuity decreased to $20 / 80$. Fundus examination revealed a pale disc and hyperpigmented retinal pigment epithelium changes in the nasal and temporal regions, corresponding to the choroidal ischemia. And the multiple Siegrist streaks are also observed (Fig. 1E). Fundus autofluorescence showed hypofluorescence in the area (Fig. 1F).

The choroid is an important structure with a highly complex vascular network and abundant blood flow. Choroidal circulation uses anastomotic channels at various levels of arterioles, venules, and capillaries. The choroid consists of multiple sectors with anastomotic arterioles. Choroidal capillaries benefit from a highly effective venous drainage system because of the many collector venules in the choroidal vascular system structure. Therefore, it is not difficult to predict the collapse of choroidal vasculature function based on the possibility of lobular occlusion following occlusion of the feeding arteriole [4]. Here, the newly formed emboli immediately after CAS may have occluded the deep choroidal feeding vessels. The choroid is mainly supplied by the posterior ciliary vessels. Because the posterior ciliary vessels are end vessels, sudden occlusion can cause choroidal ischemia. As the posterior ciliary arteries and their branches are physiologically non-anastomotic end arteries, a boundary is formed between the choroidal regions they each supply; this boundary is called the "choroidal watershed zone" [5]. Multiple emboli seemed to 

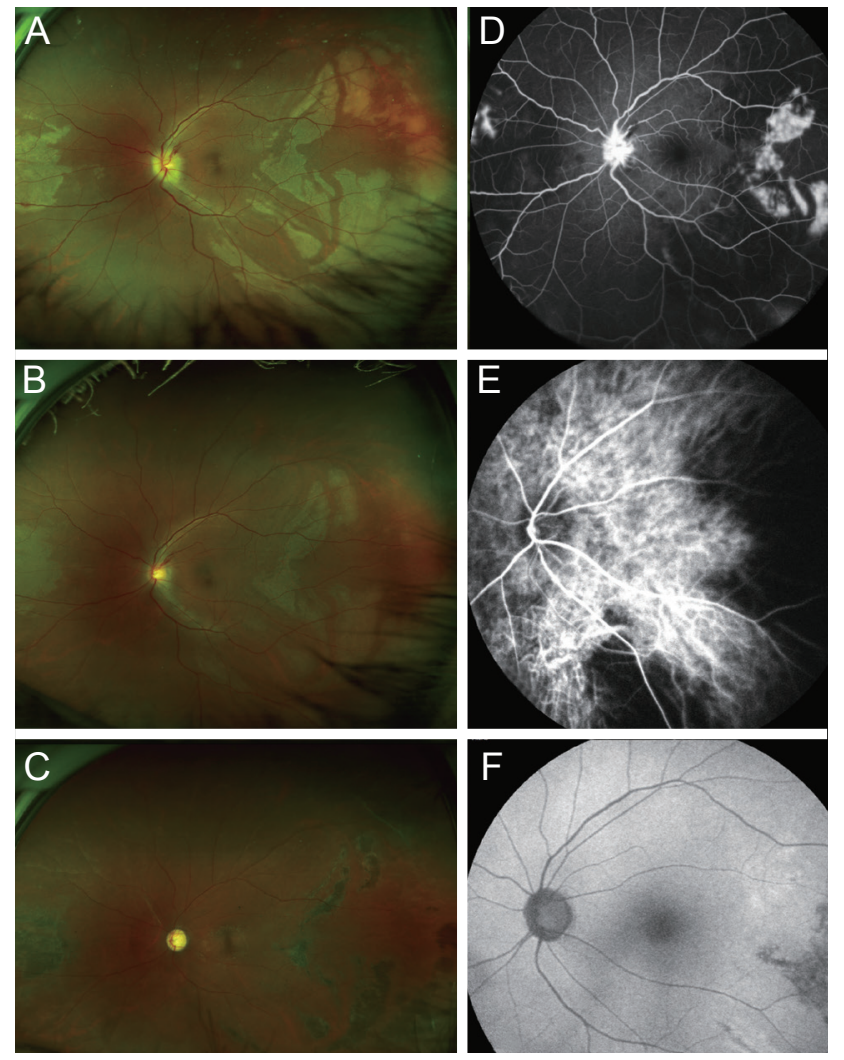

Fig. 1. Clinical photographs of this case with choroidal ischemia caused by the carotid artery stenting. (A,B) Fundus photograph and fluorescence angiography on the day of the carotid artery stenting and visual loss onset. These show an edematous disc with blurred margins and the outer retinal ischemic changes in the nasal and temporal areas (associated with the choroidal ischemia). Amalric signs and Elschnig spots are also observed. One week later, (C) fundus photograph shows less prominent outer retinal ischemic changes, and (D) indocyanine green angiography reveals a remarkable delay in the choroidal filling and a wide area of patchy choroidal filling defects. Fifteen months later, (E) fundus photograph shows hyperpigmented retinal pigment epithelium changes in the regions, corresponding to the choroidal ischemia. And the multiple Siegrist streaks are also observed. (F) Fundus autofluorescence reveals a wide area of hypofluorescence at the corresponding area. The patient provided written informed consent for publication of the research details and clinical images.

occlude the medial PCA's nasal area and the long PCA's temporal area simultaneously. Choroidal ischemia may be shortened due to the rapid recovery of choroidal blood flow by reverse flow within the choroidal veins or intra-arterial bypass channels. If sectoral atrophy is used as the sole indicator of prior choroidal hypoperfusion, many patients with choroidal ischemia will not be discovered.

In conclusion, we present a rare case of post-CAS cho- roidal ischemia considered to occur due to emboli caused by the plaque manipulation of the procedure. Therefore, a high index of clinical suspicion of choroidal ischemia should be considered in patients with visual disturbances immediately after CAS procedure.

Dae Hwan Kwon, Yu Cheol Kim, Kyung Tae Kang Department of Ophthalmology, Keimyung University Dongsan Hospital, Keimyung University School of Medicine, Daegu, Korea

E-mail (Kyung Tae Kang):kkt0604@dsmc.or.kr

\section{Conflicts of Interest}

No potential conflict of interest relevant to this article was reported.

\section{Acknowledgements}

None.

\section{Funding}

The authors received no financial support for this article.

\section{References}

1. Hayreh SS. Acute occlusive disorders of the choroidal vasculature. Int Ophthalmol 1983;6:139-48.

2. Vos JA, van Werkum MH, Bistervels JH, et al. Retinal embolization during carotid angioplasty and stenting: periprocedural data and follow-up. Cardiovasc Intervent Radiol 2010;33:714-9.

3. Barth T, Helbig H. Ischemic choroidal diseases. Klin Monbl Augenheilkd 2021;238:962-70.

4. Yoneya S, Tso MO, Shimizu K. Patterns of the choriocapillaris. A method to study the choroidal vasculature of the enucleated human eye. Int Ophthalmol 1983;6:95-9.

5. Hayreh SS. Segmental nature of the choroidal vasculature. Br J Ophthalmol 1975;59:631-48. 